\title{
Investigation of slug flow characteristics in inclined pipelines
}

\author{
J. N. E. Carneiro \& A. O. Nieckele \\ Department of Mechanical Engineering, \\ Pontificia Universidade Católica de Rio de Janeiro - PUC/Rio, RJ, Brazil
}

\begin{abstract}
In the present work a numerical analysis of the slug flow in inclined pipelines is performed with an aim to improve the understanding of slug flow characteristics over hilly-terrain section. The solution is obtained with the two-fluid model on its one-dimensional form. It consists of two sets of conservation equations of mass and linear momentum for the liquid and gas phases. The slug capturing methodology involves the numerical solution of the equations using a finite volume formulation, which is capable of naturally predicting the onset of slugging from a stratified flow regime, as well as the growth and collapse of the slugs. Flows of an oil-gas mixture in slightly inclined pipe configurations are investigated. Three types of pipelines were considered: horizontal, descending and a $V$-section pipeline. The influence of the gravity effect in average slug parameters, such as frequency, velocity and length is addressed. Qualitative comparisons with experimental observations in the literature show that the methodology seems to be able to correctly predict the effect of pipe inclination on the occurrence (or not) of the slug regime, as well as different overall slugging behaviour in $V$-section pipes if different inlet gas and liquid superficial velocities are imposed.
\end{abstract}

Keywords: slug flow, incline pipeline.

\section{Introduction}

Slug flow is a two-phase flow pattern which is characterized by a sequence of packs of liquid separated by long gas (Taylor) bubbles flowing over a liquid film inside the pipe, and is normally associated with high pressure-drops and a considerable degree of intermittency in the system. In offshore production 
systems, for example, stabilized gas and liquid flow rates are normally sought to ensure a proper operation of the plant, and separation equipments are often designed for such conditions. In this sense, previous knowledge of the flow patterns expected are of extreme importance, and if slug flow is likely to occur, it is not only important to know its mean behaviour but also the statistical details — such as the maximum slug length expected, which dictates an proper sizing of receiving equipments.

The onset of slugging in horizontal or nearly horizontal pipes is caused by two mechanisms: the natural growth of small random fluctuating disturbances at the gas-liquid interface of stratified flow (namely by a Kelvin-Helmholtz mechanism); and/or the liquid accumulation at valleys of hilly terrain pipelines with sections of different inclinations, also called terrain slugging [1-5]. Wave coalescence was also observed to be an important mechanism acting on slug formation, especially at higher gas flow rates in horizontal pipes [6-7]. Also in the $V$-section studied by Al-Safran et al. [5], this initiation mechanism was observed at relatively high gas-flow rates and low liquid flow rates, where smaller waves were unable to block the elbow.

Slug front and tail do not necessarily travel at the same velocities. A complex flow dynamics exists in which slugs may grow, collapse and merge with each other, different slugs having also different speeds [3, 8]. In this process, the mean slug length normally increases in the flow direction, because small slugs are unlikely to be stable — due to bubble wake effects - and often degenerate into long waves that are absorbed by faster slugs [3]. As a consequence of the fact that slug length, velocity and frequency are interrelated quantities, it follows that the slug frequency is likely to diminish towards the pipe ending [3,9]. As pointed out by several authors [9, 10], the spatial evolution of slugging in the pipe may require at least about $200-300$ diameters from the inlet region to achieve a developed flow. Since slugs evolve from randomly generated waves at the gas-liquid interface, the flow can also be expected to have a stochastic behaviour. In this sense, one speaks of a statistical steady state condition when the time averaged slug parameters (e.g., close to the pipe end section) do not change. Flow pattern studies [2] have shown that the pipe inclination can have a very significant effect on the stratified to slug transition, even at very small angles. It was found out that the stabilizing effect of gravity causes transition from downward stratified flow to occur at higher liquid superficial velocities (for a given gas superficial velocity), while for upward sections the transition is anticipated.

When slugs travel through a hilly terrain pipeline with different pipe inclinations, they probably undergo a change in its characteristics when moving from section to section. In addition, slugs can be generated at low elbows ( $V$-sections) or dissipated at top elbows ( $\lambda$-sections) originating a very complex pattern [5, 11]. Al-Safran et al. [5] conducted an experimental study investigating the slug flow characteristics over a hilly terrain pipe with a $V$-section, focusing on the mechanisms of slug initiation and characteristics of slugs initiated at the lower dip. An attempt is also made in order to group sets of superficial gas and liquid velocities in flow categories (superimposed on typical 
steady state flow pattern maps for the downhill upstream pipe), according to the influence of the $V$-section on the characteristics of developed slug flow upstream of the downward pipe.

As described by Issa and Kempf [8], transient models in the context of pipeline slugging usually solve the Two-Fluid Model equations [12] in its transient one-dimensional version and can be grouped into three categories: empirical slug specification, slug tracking and slug capturing. In contrast to the other ones, a key feature of the slug capturing methodology is the capability of predicting the evolution from stratified to slug flow in a natural manner, i.e., there is no need to incorporate any transition criteria assuming that slugs were generated somehow in the pipe (e.g., by use flow pattern maps). This means that the natural outcome of the solution of the equation system can be either the maintenance of stratified flow in the pipe, or the change in regime if conditions are such that slugs develop in the system. If transition occurs, slugs may grow or decay as they travel downstream in the pipe, and no empirical correlations for slug parameters need to be specified. Thus, the set of equations is maintained even when the regime changes and the slug dynamics is an automatic consequence of the solution of the system.

At the present work, the Two Fluid Model is employed to predict the slug formation in horizontal and slightly inclined pipeline. To validate the methodology, comparison is performed with the experimental data of Al-Safran et al. [5].

\section{Mathematical modelling}

The mathematical model selected is based on the slug capturing technique, in which the slug formation is predicted as a result of a natural and automatic growth of the hydrodynamic instabilities $[8,13]$. Both stratified and slug pattern are modeled by the same set of conservation equations based on the Two-Fluid Model. Additionally, closure relations are also included. The liquid is considered as incompressible, while the gas follows the ideal gas law, $\rho_{G}=P /(R T)$, where $R$ is the gas constant and $T$ is its temperature, which was considered here as constant. Pressure $P$ was considered constant long the cross section, being the same, for the liquid $P_{L}$, gas $P_{G}$ and interface $\left(P=P_{G}=P_{L}\right)$. Additionally, it was assumed that there is no mass transfer between phases. The governing mass and momentum equations in the conservative form can be written as

$$
\begin{aligned}
& \frac{\partial\left(\rho_{G} \alpha_{G}\right)}{\partial t}+\frac{\partial\left(\rho_{G} \alpha_{G} u_{G}\right)}{\partial x}=0 ; \frac{\partial\left(\rho_{L} \alpha_{L}\right)}{\partial t}+\frac{\partial\left(\rho_{L} \alpha_{L} u_{L}\right)}{\partial x}=0, \\
& \frac{\partial\left(\rho_{G} \alpha_{G} u_{G}\right)}{\partial t}+\frac{\partial\left(\rho_{G} \alpha_{G} u_{G}^{2}\right)}{\partial x}=-\alpha_{G} \frac{\partial P}{\partial x}-\rho_{G} \alpha_{G} g \sin (\beta)- \\
& -\rho_{G} \alpha_{G} g \frac{\partial h}{\partial x} \cos (\beta)-F_{G w}-F_{i},
\end{aligned}
$$




$$
\begin{aligned}
& \frac{\partial\left(\rho_{L} \alpha_{L} u_{L}\right)}{\partial t}+\frac{\partial\left(\rho_{L} \alpha_{L} u_{L}^{2}\right)}{\partial x}=-\alpha_{L} \frac{\partial P}{\partial x}-\rho_{L} \alpha_{L} g \sin (\beta)- \\
& -\rho_{L} \alpha_{L} g \frac{\partial h}{\partial x} \cos (\beta)-F_{L w}+F_{i},
\end{aligned}
$$

where $\alpha_{G}+\alpha_{L}=1$. The subscripts $G, L$, and $i$ concern the gas, liquid phases and interface, respectively. The axial coordinate is $x, \rho$ and $\alpha$ are the density and volumetric fraction, $u$ is the velocity. The pipeline inclination is $\beta, h$ is the liquid level inside the pipe, and $g$ is the gravity acceleration. The third term on the right side of eqs. (2) and (3) are related with the hydrostatic pressure at the gas and liquid, respectively. The term $F=\tau S / A$ is the friction force per unit volume between each phase and the wall and between the phases (at the interface), where $\tau$ is the shear stress, $S$ is the phase perimeter and $A$ is the pipe cross section area.

The shear stress is $\tau=f \rho\left|u_{r}\right| u_{r} / 2$, where $u_{r}$ is the relative velocity between the liquid and wall, the gas and wall, or gas and liquid. Closure relations are needed to determine the friction factor $f$.

The flow was considered in the laminar regime, when the Reynolds number $\mathbf{R e}$, was smaller the $2100\left(\mathbf{R e}_{G} ; \mathbf{R e}_{i}\right.$ and $\mathbf{R e}_{L}$ for the gas, interface and liquid, respectively). The Hagen-Poiseulle formulas were employed for the gas-wall and interface laminar friction factor and the correlation of Hand [14] for the liquid-wall laminar friction factor, while the correlation of Taitel and Dukler [1] was adopted for the turbulent gas-wall and interface friction factor and the Spedding and Hand [15] correlation for turbulent the liquid-wall friction

$$
\begin{array}{r}
\text { laminar: } f_{G}=\frac{16}{\mathbf{R e}_{G}}, \quad f_{i}=\frac{16}{\mathbf{R e}_{i}}, \quad f_{L}=\frac{24}{\mathbf{R e}_{L}^{s}} \\
\text { turbulent: } f_{G}=\frac{0.046}{\mathbf{R e}_{G}^{0.25}}, \quad f_{i}=\frac{0.046}{\mathbf{R e}_{i}^{0.25}}, \quad f_{L}=\frac{0.0262}{\left(\alpha_{L} \mathbf{R e}_{L}^{s}\right)^{0.139}},
\end{array}
$$

where $\alpha_{L}$ is the hold-up (liquid volumetric fraction). The Reynolds numbers are defined as

$$
\begin{gathered}
\operatorname{Re}_{G}=\frac{4 A_{G} u_{G} \rho_{G}}{\left(S_{G}+S_{i}\right) \mu_{G}}, \quad \mathbf{R e}_{i}=\frac{4 A_{G}\left|u_{G}-u_{L}\right| \rho_{G}}{\left(S_{G}+S_{i}\right) \mu_{G}}, \\
\operatorname{Re}_{L}=\frac{4 A_{L} u_{L} \rho_{L}}{S_{L} \mu_{L}}, \quad \mathbf{R e}_{L}^{s}=\frac{\rho_{L} U_{S} D}{\mu_{L}}
\end{gathered}
$$

where $\mu$ is the absolute viscosity and $D$ is the pipe diameter. The last Reynolds in eq. (5) is based on the liquid superficial velocity, i.e., the ration of the liquid volume flow rate to the total cross section area of the pipe $U s_{L}=Q_{L} / A=\alpha_{L} u_{L}$. 
The geometric parameters such as gas and liquid areas $\left(A_{G}, A_{L}\right)$, wetted perimeters $\left(S_{G}, S_{L}\right)$, and interface width $S_{i}$ where obtained from the liquid height $h[13]$.

\section{Numerical method}

The conservation equations were discretized by the Finite Volume Method [16]. A staggered mesh was employed, with both phases' velocities stores at the control volume faces and all other variables at the central point. The interpolation scheme upwind and the implicit Euler scheme were selected to evaluate the space and time derivates, respectively. The set of resulting equations consists of two momentum equations, one pressure equation (global mass conservation) and one gas volumetric fraction (gas mass conservation). These equations were solved sequentially, through an iterative method [13].

The time step was specified to guarantee a Courant number equal to 0.5 [8], therefore, the time step was obtained from $\Delta t=0.5 \Delta x_{i}\left|u_{\max }\right|$, where $u_{\max }$ is the maximum velocity in the domain. For each time step, due to the non linearities of the problem, the sequence of conservation equations were solved in an iterative process, until convergence was obtained, that is, until the residue of all equations became smaller than 0.0001 .

\section{Results}

At the present work a numerical analysis of the slug flow in inclined pipelines is performed aiming to improve the understanding of slug flow characteristics over hilly-terrain section. Three types of pipelines are investigated: horizontal, descending and a $V$-section pipeline.

A $V$-section pipeline was defined based on the experimental work of AlSafran et al. [5]. The pipeline consists of descending and ascending sections with length of $21.34 \mathrm{~m}$, and inclination of $\beta=-1.93^{\circ}$ and $\beta=+1.93^{\circ}$ in relation to the horizontal direction as illustrated in Fig. 1a. To guarantee a smooth transition between the downward and upward sections, a small horizontal section of $0.3 \mathrm{~m}$ joining the two parts was added. The pipeline diameter is equal to $D=0.0508 \mathrm{~m}$. The total length of the pipelines is equal to $L=42.98 \mathrm{~m}$.

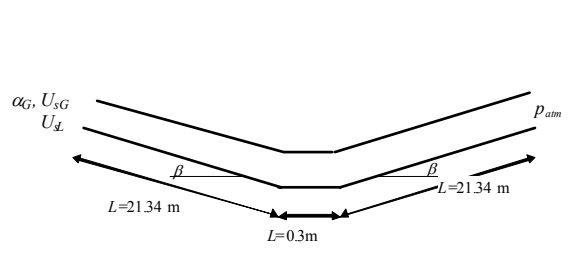

(a)

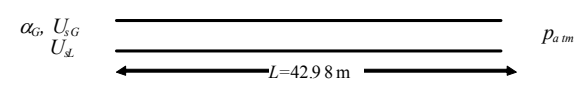

(b)

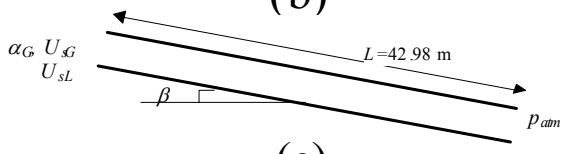

(c)

Figure 1: Configurations considered: (a) V-section pipeline; (b) horizontal pipeline (c) slightly inclined pipeline. 
To investigate the effect of small inclinations in the slug flow parameters, the same conditions were tested in a horizontal pipeline, Fig. 1b, and in a pipeline with a downward inclination of $\beta=-1.93^{\circ}$, Fig. 1c. All pipelines have the same total length $L$.

The same two-phase fluid mixture (air and oil) employed by [5] was defined. The air was considered as ideal gas with gas constant $R=287 \mathrm{~N} \mathrm{~m} /(\mathrm{kg} \mathrm{K})$, with molecular viscosity of $\mu_{G}=1.796 \times 10^{-5} \mathrm{~Pa}$ s. The oil density was $\rho_{L}=890.6$ $\mathrm{kg} / \mathrm{m}^{3}$, and molecular viscosity a $\mu_{L}=1.02 \times 10^{-2} \mathrm{~Pa}$.s. The inlet liquid holdup $\alpha_{L}$ was defined as $0.4\left(\alpha_{G}=0.6\right)$ and a constant atmospheric pressure $p_{\text {atm }}$ was kept at outlet.

The initial condition was defined as a stratified steady state flow, that is, constant liquid height along the pipeline, with constant liquid and gas velocities, and pressure distribution obtained by solving the momentum conservation equation, considering equilibrium stratified flow.

The flow field is determined based on the two-fluid model $[8,13]$ by the solution of the momentum conservation equations for each phase, continuity of the gaseous phase and total mass conservation.

Two situations were considered, classified as Category I and II. The gas and liquid superficial velocities were defined as $U s_{L}=0.6 \mathrm{~m} / \mathrm{s}$ and $U s_{G}=0.64 \mathrm{~m} / \mathrm{s}$ for Category I and $U s_{L}=1.22 \mathrm{~m} / \mathrm{s}$ and $U s_{G}=1.3 \mathrm{~m} / \mathrm{s}$ for Category II.

Figures 2 illustrates successive liquid hold-up profiles along the pipeline in time for the horizontal and descending cases for Category I, while Fig. 3 corresponds to Category II. The liquid hold-up profile in time for the $V$-section pipeline is shown in Fig. 4 for both Categories.

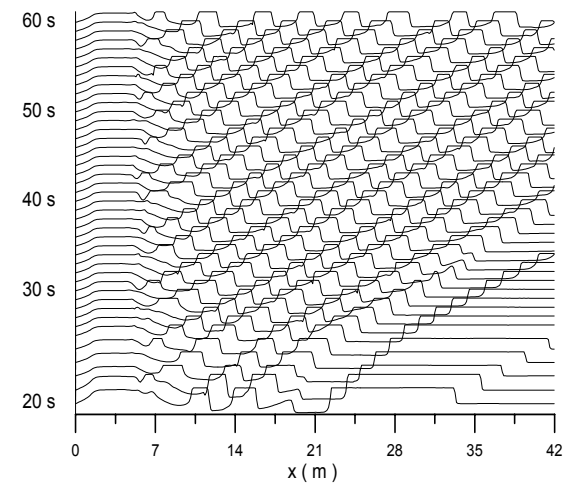

(a)

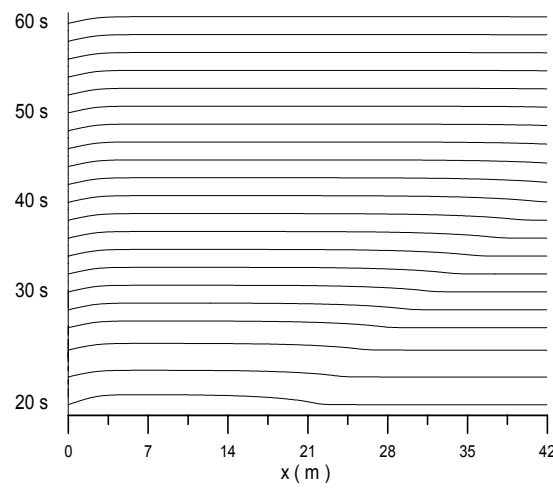

(b)

Figure 2: $\quad$ Successive hold-up profiles in time. Category I: $U s_{L}=0.60 \mathrm{~m} / \mathrm{s}, U s_{G}$ $=0.64 \mathrm{~m} / \mathrm{s}$ : (a) horizontal, (b) descending.

As it can be seen in Fig. 2, for Category I, slug pattern is observed in the horizontal pipeline, Fig. 2a, but it is not observed in the descending pipeline, Fig. $2 b$, due to the gravity stabilizing effect which inhibits small perturbations to 
grow at the interface, inducing the slug. For the $V$-section pipeline shown in Fig. $4 \mathrm{a}$, the slug flow is formed by the accumulation of liquid at the dip. This was the same behavior observed experimentally in [5], for the same superficial velocities.

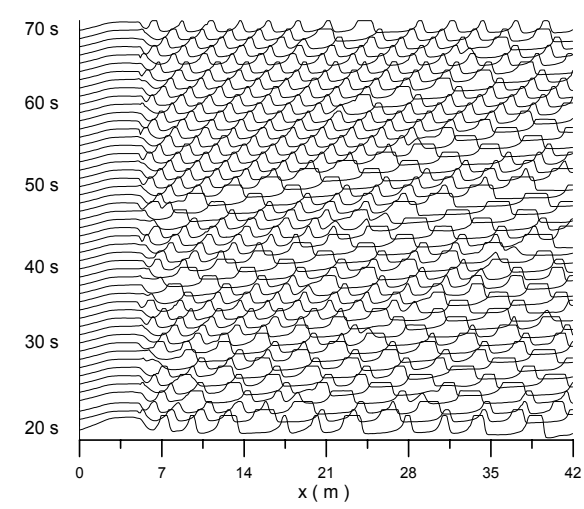

(a)

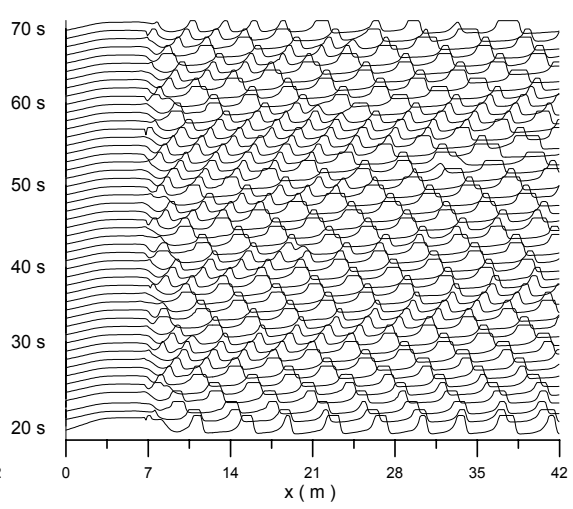

(b)

Figure 3: Successive hold-up profiles in time. Category II: $U s_{L}=1.22 \mathrm{~m} / \mathrm{s}$, $U s_{G}=1.30 \mathrm{~m} / \mathrm{s}$ : (a) horizontal, (b) descending.

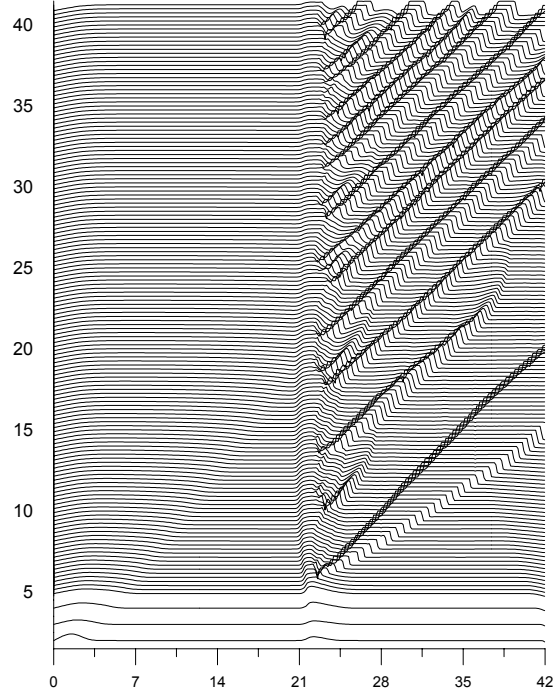

(a)

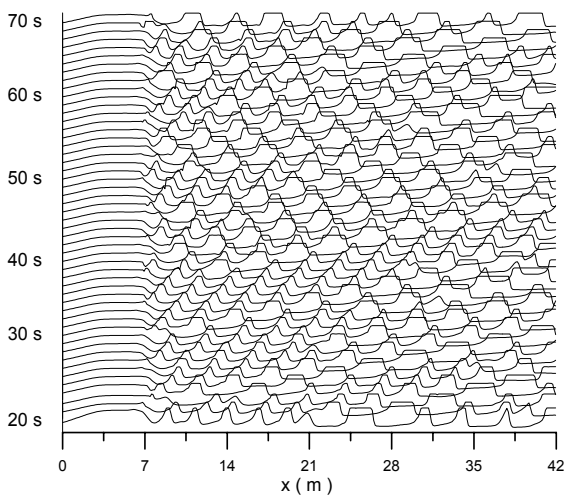

(b)

Figure 4: Successive hold-up profiles in time. V-section pipeline: (a) Category I: $U s_{G}=0.64 \mathrm{~m} / \mathrm{s}, U s_{L}=0.60 \mathrm{~m} / \mathrm{s}$, (b) Category II: $U s_{L}=1.22 \mathrm{~m} / \mathrm{s}, U s_{G}=1.30 \mathrm{~m} / \mathrm{s}$.

The hold-up profiles in time for Category II, shown in Figs. 3 and 4b, show that, as oppose to the Category I case, it can be seen that due to same slug formation mechanism, the slugs are formed approximately at $7 \mathrm{~m}$ from the inlet 
for all cases. The effect of gravity is to delay just a little the slug formation. It can also be seen, that due to the high frequency, there is not enough time to occur liquid accumulation at the dip of the $V$-section pipeline, therefore, there are no additional slugs being formed. Once again, these observations agree with the experiments of [5].

For the horizontal and slightly inclined pipeline a pattern map was built based on the studies of Taitel and Dukler [1] and Barnea and Taitel [17]. The predictions obtained with the present work agreed perfectly with the pattern maps.

The slug translation velocity $U_{t}$, length $L_{s}$ and frequency $v_{s}$ were determined for the three pipelines configurations. The mean slug translation velocity $U_{t}$ is inferred by the dimensionless parameter $C_{o}$, based on the same correlation employed in [8],

$$
\begin{aligned}
& U_{t}=C_{o} U_{M}+U_{d}
\end{aligned}
$$

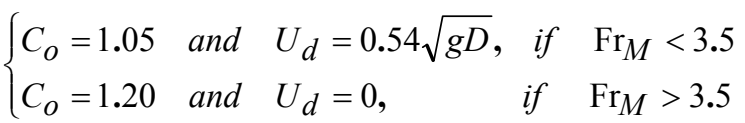

where the mixture velocity $U_{M}$ is equal to the sum of the inlet liquid and gas superficial velocities, $U_{M}=U s_{L}+U s_{G}$. The Froude number $\mathbf{F r}_{M}$ is based on the mixture velocity as

$$
\operatorname{Fr}_{M}=U_{M} / \sqrt{g D}
$$

The slug parameters corresponding to $x=37 \mathrm{~m}$ are shown in Table 1. It can be seen that for the first category the liquid accumulation at the dip leads to a superior frequency for the $V$-section than the horizontal case, since the length is smaller, once the velocities are similar. This tendency was also experimentally observed by [5]. It should be mentioned here, that it was only possible to perform a qualitative comparison, since the data of [5] were not available due to proprietary restriction.

Table 1: Slugs characteristics.

\begin{tabular}{|c|c|c|c|c|c|c|}
\hline & \multicolumn{2}{|c|}{$\boldsymbol{C}_{\boldsymbol{o}}$} & \multicolumn{2}{c|}{$\boldsymbol{v}_{\boldsymbol{s}}(\mathbf{1} / \mathbf{s})$} & \multicolumn{2}{c|}{$\boldsymbol{L}_{\boldsymbol{s}} \boldsymbol{D}$} \\
\hline Category & I & II & I & II & I & II \\
\hline Horizontal & 1.25 & 1.40 & 0.33 & 0.95 & 42.6 & 13.9 \\
\hline Descending & - & 1.39 & - & 0.94 & - & 13.5 \\
\hline V-section & 1.23 & 1.37 & 0.38 & 0.83 & 29.6 & 21.3 \\
\hline
\end{tabular}

Table 1 shows that a slightly higher velocity is found for Category II. The slug length of the horizontal and descending cases differed by $5 \%$, and the frequency was approximately constant. However for the $V$-section the slug length was $58 \%$ larger, leading to a $14 \%$ reduction of the frequency in relation to other two cases. The increase in the length is due to the accumulation of liquid at the 
dip, which did not induce the formation of new slugs, but increased its length. Further the liquid velocity at the ascending section is smaller, what also contributed to increase the slug length.

Figure 5 shows the average slug length along the pipelines, for Category II. It can be seen that mean length is approximately the same for the horizontal and descending case, were the gravity effect is negligible due to the high velocities. Larger slug lengths are observed along the V-section pipeline, especially at the ascending section due to the accumulation of liquid at the dip as described previously. It can be clearly seen that slug length distribution changes across a symmetrical pipeline, since the gravity effect is not symmetrical.

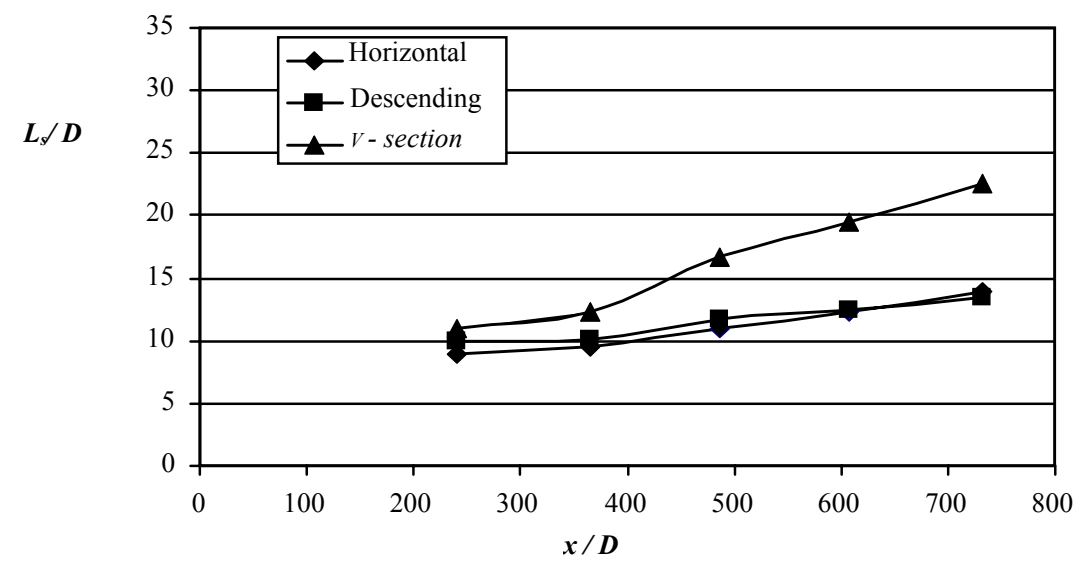

Figure 5: Average slug length along horizontal, descending and $V$-section pipelines: $U s_{L}=1.22 \mathrm{~m} / \mathrm{s}$ and $U s_{G}=1.3 \mathrm{~m} / \mathrm{s}$.

\section{Final remarks}

The Two Fluid Model was employed to predict the slug formation along horizontal, slightly inclined and $V$-section pipelines. The results obtained qualitatively agreed with the experimental data of [5]. The flow can be classified in different Categories, depending in the gravity influence to damp the slug formation. The accumulation of liquid in lower sections of the pipeline can increase not only the size of the slug, but also its velocity.

\section{Acknowledgements}

The authors thank the Brazilian Research Council, CNPq for the support awarded to this work.

\section{References}

[1] Taitel, Y. \& Dukler, A.E., A model for predicting flow regime transitions in horizontal and near horizontal pipes, AIChE Journal, 22, pp. 47-55, 1976. 
[2] Barnea, D., A unified model for predicting flow-pattern transitions for the whole range of pipe inclinations, International Journal of Multiphase Flow, 13, pp. 1-12, 1987.

[3] Taitel, Y. \& Barnea, D., Two-phase slug flow, Advances in Heat Transfer, 20, pp. 83-132, 1990.

[4] Fabre, J. \& Liné, A., Modeling of two-phase slug flow, Annual Review of Fluid Mechanics, 24, pp. 21-46, 1992.

[5] Al-Safran, E., Sarica, C., Zhang, H.Q. \& Brill, J., Investigation of slug flow characteristics in the valley of a hilly terrain pipeline, International Journal of Multiphase Flow, 31, 337-357, 2005.

[6] Lin, Y.P. \& Hanratty, T. J., Prediction of the initiation of slugs with the linear stability theory, International Journal of Multiphase Flow, 12, pp. 79-98, 1987.

[7] Woods, B. D., Fan, Z. \& Hanratty, T. J., Frequency and development of slugs in a horizontal pipe at large liquid flows, International Journal of Multiphase Flow, 32, pp. 902-925, 2006.

[8] Issa, R. I. \& Kempf, M. H. W., Simulation of slug flow in horizontal and nearly horizontal pipes with the two fluid model, International Journal of Multiphase Flow, 29, 69-95, 2003.

[9] Tronconi, E., Prediction of slug frequency in horizontal two-phase slug flow, AIChE Journal, 36, pp. 701-709, 1990.

[10] Barnea, D. \& Taitel, Y., A model for slug length distribution in gas-liquid slug flow, International Journal of Multiphase Flow, 19, pp. 829-838, 1993.

[11] Zheng, G., Brill, J. P. \& Taitel, Y. Slug flow behavior in a hilly terrain pipeline, International Journal of Multiphase Flow, 20, pp. 63-79, 1994.

[12] Ishii, M., Thermo-Fluid Dynamic Theory of Two-Phase Flow, Eyrolles, Paris, 1975.

[13] Carneiro, J. N. E., Ortega, A. J., Nieckele, A. O., Influence of the Interfacial Pressure Jump Condition on the Simulation of Horizontal TwoPhase Slug Flows Using the Two-Fluid Model, Proceedings of $3^{\text {rd }}$ International Conference on Computational Methods in Multiphase Flow 2005, Portland, Maine, USA, pp. xxx-xxx, 2005.

[14] Hand, N.P. Gas-liquid co-current flow in a horizontal pipe, Ph.D. Thesis, Queen`s University Belfast, 1991.

[15] Spedding, P.L.; Hand, N.P., Prediction in stratified gas-liquid co-current flow in horizontal pipelines, International Journal Heat Mass Transfer, 40, pp. 1923-1935, 1997.

[16] Patankar, S.V., Numerical Heat Transfer and Fluid Flow, Hemisphere Publishing Corporation, 1980.

[17] Barnea, D. \& Y. Taitel, Y., Interfacial and structural stability of separated flow, International Journal of Multiphase Flow, 19, pp. 387-414, 1994. 